\title{
Pediatric infectious diseases by J.M. Bergelson, S.S. Shah and T.E. Zaoutis
}

\author{
Sergio Fanconi
}

Received: 26 March 2009 /Accepted: 29 March 2009/Published online: 27 April 2009

(C) Springer-Verlag 2009

This new volume of the series The Requisites in Pediatrics is a multi-author book (55 authors mainly from the USA) edited by three infectious diseases specialists of the Children's Hospital of Philadelphia, PA, dedicated to students, residents, family practitioners, general pediatricians, and nurse practitioners. It is not a reference book for specialists in infectious diseases but covers all practically important fields of pediatric infectious diseases. The major part is dedicated to diagnosis and treatment, making it especially interesting for daily practice and primary care in the hospital and outside the hospital. As infectious problems are the most frequent pathologies encountered in the pediatric practice, this book is an excellent preparation for general pediatrics.
The contents are well organized discussing in a synthesized manner (a) the basic microbiology and antimicrobial therapies, (b) the most common infections including the problem of the febrile child, (c) special problems, for example the neonate or the immunocompromized patient or HIV infection, etc., and (d) prevention and infection control. This easy-to-read book is an up-to-date book summarizing the authors experience as well as the evidence in the literature. It concentrates on what is important in each domain. The tables and figures as well as the summary of the major points at the end of each chapter are particularly useful, and the suggested readings, which are limited to not more than 20 to 30 references, are extremely helpful if the reader is looking for more information or has a special interest.

S. Fanconi $(\bowtie)$

Chef Département Médico-Chirurgical de Pédiatrie Centre

Hospitalier Universitaire Vaudois (CHUV),

CH-1011 Lausanne, Switzerland

e-mail: sergio.fanconi@chuv.ch 